\title{
EXPERIENCIA DE PROFESIONALES DE ENFERMERÍA QUE SE DESEMPEÑAN EN UN RECINTO PENITENCIARIO DEL NORTE DE CHILE
}

\author{
EXPERIENCE OF NURSING PROFESSIONALS WORKING IN A \\ CORRECTIONAL INSTITUTION IN THE NORTH OF CHILE
}

\section{EXPERIÊNCIA DE PROFISSIONAIS DE ENFERMAGEM QUE TRABALHAM EM INSTALAÇÓES PENITENCIÁRIAS NO NORTE DO CHILE}

\author{
Valentina Burgos Flores* \\ Dayana Páez Olivares** \\ Camila Vio CháVez ${ }^{* * *}$ \\ Mauricio Díaz Araya**** \\ Rosa VEGA FLORES ${ }^{* * * * *}$
}

\begin{abstract}
RESUMEN
Durante las últimas décadas se han producido numerosos avances en Enfermería, sin embargo, las experiencias de enfermeros que desempeńan su labor en recintos penitenciarios continúan siendo una realidad desconocida. Objetivo: Describir las experiencias profesionales de enfermeros/as que trabajan en un recinto penitenciario en el norte de Chile. Material y Método: Estudio cualitativo, exploratorio y descriptivo centrado en relatos de la experiencia profesional; participaron 6 enfermeras(os). El estudio, aprobado por un comité de ética acreditado, se realizó entre agosto y octubre de 2018, utilizando la entrevista semiestructurada. Se consideraron criterios de calidad y rigor científico. Resultados: El centro de salud penitenciario cuenta con una gran infraestructura y equipo multidisciplinario para brindar atención de salud a la población, donde su organización se realiza en base a la demanda existente, basada en un conjunto de reglamentos y normas que regulan el funcionamiento de la Institución. Se describe que las áreas de enfermería más desarrolladas son el quehacer asistencial y de gestión, en donde el rol de enfermería es fundamental al interior de los equipos de salud. Así mismo, los/as enfermeros/
\end{abstract}

\footnotetext{
*Enfermera, Unidad de cuidados Intensivos, Clínica Indisa, Santiago, Chile. ORCID: http://orcid.org/0000.0002-8374-371x Email: valentinaburgosf@gmail.com

**Enfermera, Unidad de tratamiento intensivo, Hospital San Juan de Dios, La Serena, Chile. ORCID: http://orcid.org/00016142-1931 Email: dayanapaez007@gmail.com

***Enfermera, Unidad de cuidados Intensivos pediátricos, Hospital Carlos Van Buren, Valparaíso, Chile. ORCID: http://orcid. org/0000.0003-0147-9474 Email: camilaviochavez@gmail.com

****Psicólogo, Magíster en Psicología mención Comunitaria, Universidad Católica del Norte, Chile. ORCID: http://orcid .org/ 0000.0002-2380-0196 Email: mdiazg@ucn.cl.

***** Doctora en Enfermería, Magíster en Educación, Facultad de Medicina, Universidad Católica del Norte, Coquimbo, Chile. ORCID: http://orcid.org/0002-1576-1705 Email: rvega@ucn.cl. Autora de correspondencia.
} 
as describen este campo laboral como una gran oportunidad de aprendizaje y manifiestan sentir un grato clima laboral, pese al estrés propio del contexto. Conclusión: El recinto penitenciario brinda a los profesionales de enfermería un lugar versátil de trabajo, que a su vez permite desarrollar capacidades de liderazgo y autonomía, difíciles de encontrar en otros puestos laborales; los mismos profesionales lo consideran un campo laboral muy recomendado para futuros colegas que busquen nuevos desafíos.

Palabras clave: Enfermeras y Enfermeros; Prisiones; Rol de la enfermera; Atención de Enfermería.

\section{ABSTRACT}

During the last decades there have been several advances in nursing, however the experiences of nurses who work in prisons are still an unknown reality. Objective: To describe the professional experiences of nurses who work in a prison in the north of Chile. Material and Method: Qualitative, descriptive and exploratory study focused on the accounts of professional experience of 6 nurses. The research was approved by an accredited ethics committee and was carried out between August and October 2018, using a semi-structured interview. Quality criteria and scientific rigor were considered. Results: The prison health center has a large infrastructure and a multidisciplinary team to provide health care to the inmates, where organization is based on existing demand, based on a set of regulations and norms that regulate the operation of the correctional institution. It is noted that the most developed areas of nursing are care and management, where the role of nursing is considered essential within the health teams. Additionally, nurses describe this work field as a great learning opportunity and express a positive working environment despite the stressful context. Conclusion: Working in a prison offers nurses a versatile workplace that also allows them to develop leadership and autonomy skills, which are difficult to find in other jobs. Many nursing professionals recommend this work field for future colleagues seeking new challenges.

Key words: Nurses; Prisons; Nurse's role; Nursing care.

\section{RESUMO}

Nas últimas décadas ocorreram inúmeros avanços na Enfermagem, porém, as vivências dos enfermeiros que realizam seu trabalho em instalaçóes penitenciárias continuam sendo uma realidade desconhecida. Objetivo: Descrever as experiências profissionais de enfermeiras/os que atuam em uma penitenciária no norte do Chile. Material e Método: Estudo qualitativo, exploratório e descritivo com foco em relatos de experiência profissional; participaram 6 enfermeiras/os. O estudo, aprovado por um comitê de ética credenciado, foi realizado entre agosto e outubro de 2018, por meio de entrevista semiestruturada. Critérios de qualidade e rigor científico foram considerados. Resultados: $\mathrm{O}$ centro de saúde penitenciário possui ampla infraestrutura e equipe multiprofissional para atendimento à saúde da população, onde sua organização é realizada com base na demanda existente, a partir de um conjunto de regulamentos e normas que estabelecem o funcionamento da Instituição. Descreve-se que as áreas mais desenvolvidas da enfermagem são o trabalho assistencial e gerencial, onde a atuação da enfermagem é fundamental dentro das equipes de saúde. Da mesma forma, as/os enfermeiras/ os descrevem este campo de trabalho como uma grande oportunidade de aprendizagem e afirmam que sentem um ambiente de trabalho agradável, apesar do estresse inerente ao contexto. Conclusão: $\mathrm{O}$ estabelecimento penitenciário proporciona aos profissionais de enfermagem um ambiente de trabalho versátil, que por sua vez lhes permite desenvolver habilidades de liderança e autonomia, difíceis de encontrar em outros empregos; os mesmos profissionais consideram-no um campo de trabalho altamente recomendado para futuros colegas que procuram novos desafios.

Palavras-chave: Enfermeiras e enfermeiros; Prisóes; Papel da enfermeira; Cuidados de enfermagem.

Fecha de recepción: 31/12/2019

Fecha de aceptación: 12/03/2021 


\section{INTRODUCCIÓN}

El profesional de Enfermería, a nivel internacional y Latinoamérica, se ha ido adaptando a diferentes contextos laborales y así surge la demanda de su presencia en los sistemas penales o recintos penitenciarios (RP). Los equipos de salud perciben la importancia de la presencia del enfermero(a) en las cárceles para optimizar el acceso de las personas a las acciones e intervenciones de cuidados en salud, fundamentalmente para promover, mantener y recuperar la salud de las personas que se encuentran privadas de libertad ${ }^{(1,2)}$. En Chile la atención sanitaria en los RP presenta características particulares y diferentes, según la percepción de internos que consideran que el acceso a la atención de salud es difícil ${ }^{(3)}$.

La Enfermería penitenciaria es una especialidad única, contempla aspectos de la salud relacionados con la custodia y los cuidados; la labor que desempeña el profesional en los RP presenta características específicas, porque cuida a personas privadas de libertad, en un ambiente hostil y bajo influencias legales y/o regimentales ${ }^{(4)}$.

La especialización penitenciaria, según la American Nurses Association (ANA), es un compendio de salud pública, urgencias, salud comunitaria, salud ocupacional y atención ambulatoria, donde el trabajo de las/os enfermeras(os) consiste en cuidar a personas con problemas de salud complejos ${ }^{(5,6)}$. En el transcurso del tiempo los profesionales de enfermería que trabajan en entornos penitenciarios han desarrollado habilidades específicas para poder brindar la atención adecuada a sus pacientes y estas incluyen la adaptación, tanto al entorno penitenciario, como a la cultura penitenciaria ${ }^{(7)}$.

Según lo anterior, la labor que desempeña el profesional de enfermería en los RP se diferencia en la atención que brinda en el medio externo ${ }^{(8)}$, porque, en este contexto, debe enfrentar grandes retos para desarrollar cuidados en las mejores condiciones $^{(9)}$. Debe garantizar bienestar y seguridad, junto con preservar la salud de las personas, independientemente de su condición ${ }^{(10)}$, la que debe ser equivalente a la de las personas que se encuentran en libertad, considerando que todos los privados de libertad también son seres humanos con derechos ciudadanos ${ }^{(11)}$.

Es importante considerar la influencia que este entorno juega en todos los agentes involucrados, y más específicamente en el profesional de enfermería, que brinda una diversidad de cuidados. Por ello, las/os enfermeras/os deben capacitarse en áreas específicas como salud mental, abuso de drogas, emergencias, atención primaria y salud pública, así como en aspectos legales y regulatorios ${ }^{(12)}$.

En los RP se concentran condicionantes específicas de salud, tales como: drogodependencias, enfermedades infecciosas (VIH/Sida, tuberculosis $^{(13)}$, hepatitis, infecciones de transmisión sexual), trastornos mentales, así como factores que derivan de la atención en situaciones límites, condiciones que no difieren de la experiencia internacional ${ }^{(14,15)}$.

Inserto en este contexto, el profesional de enfermería brinda a los pacientes educación, realiza exámenes físicos, distribuye medicamentos, asegura el cumplimiento y continuidad del tratamiento, da primeros auxilios, realiza exámenes de detección, capacita al personal y brinda cuidados postoperatorios, además de entregar atención de emergencia $^{(16)}$. La administración de los tratamientos se considera una actividad relevante en el papel de las/os enfermeras/os en un espacio confinado, porque está marcado por actividades de vigilancia, en particular en el umbral de la celda. Por ello, en algunas oportunidades la relación terapéutica es difícil de establecer y ello impide, en parte, el despliegue de los cuidados de enfermería ${ }^{(17)}$.

La Institución que debe velar por esta vigilancia y asistencia de la población penal es Gendarmería de Chile $(\mathrm{GENCHI})^{(18,19)}$ y la atención de salud que se brinda a las personas privadas de libertad está orientada por políticas emanadas de la autoridad sanitaria (Ministerio de Salud, Servicios de Salud), considerando aspectos administrativos, técnicos, protocolos, procedimientos de traslados y derivaciones, autorización sanitaria de unidades de salud, trabajo intersectorial y sistema de registros y estadísticos ${ }^{(20)}$. Para ofrecer estos servicios existe, en los RP, un equipo de salud multidisciplinario constituido por profesionales médicos, enfermeras/ os, asistentes sociales, abogados, psicólogos, nutricionistas y técnicos en enfermería ${ }^{(20)}$.

El profesional de enfermería tiene un rol fundamental, asociado al desempeño en la entrega de cuidados específicos, sustentado en conocimientos propios de la disciplina para brindar atención de salud a la población ${ }^{(21,22)}$. Las/os enfermeras/os brindan cuidados holísticos, con una actitud crítica y reflexiva, es decir, integran competencias de 
pensamiento crítico y cuestionamiento científico con principios éticos, como base para establecer juicios profesionales. Según lo anterior, basa la práctica de los cuidados en la evidencia, de forma autónoma y colaborativa, porque se vincula a las fuentes de información ${ }^{(23-25)}$ centradas en las necesidades de las personas, considerando aspectos éticos y legales de la profesión, de acuerdo a las características del sistema penitenciario.

Las personas recluidas, independientemente de la naturaleza de su transgresión, conservan el derecho a disfrutar de los más altos estándares de atención de salud, ya que preservan todos los derechos inherentes a su ciudadanía, y la atención de enfermería contribuye a ese bienestar de forma integral ${ }^{(20)}$.

De acuerdo a los antecedentes presentados y aunque en las últimas décadas se han producido numerosos avances en enfermería, las experiencias de enfermeros que desempeñan su labor en recintos penitenciarios continúan siendo una realidad desconocida. Por ello, este estudio tuvo como propósito describir las experiencias profesionales de enfermeras/os que se desarrollan en RP del norte de Chile.

\section{MATERIAL Y MÉTODO}

Estudio de tipo cualitativo, de diseño exploratorio descriptivo, con enfoque biográfico, que permitió explorar las experiencias vividas por las/los enfermeras/os que se encuentran trabajando en un recinto penitenciario del norte de Chile ${ }^{(26,27)}$. La investigación se realizó entre los meses de agosto a octubre del año 2018; se seleccionó el recinto penitenciario más cercano al equipo de investigación, siendo este criterio fundamental para el desarrollo del trabajo de campo, ya que la proximidad al fenómeno de investigación requería un proceso de inmersión constante en el campo de estudio ${ }^{(28)}$. Otro criterio fue el contar con las redes de contacto profesional que el equipo de investigación logró fortalecer, ya que ingresar a un recinto, con estas características, requiere de protocolos, entrevistas y autorizaciones formales de la dirección de los RP. Ambos criterios se fundamentan en el tipo de muestreo intencionado o por conveniencia, donde el equipo de investigación decide según sus alcances y facilidades del campo a investigar ${ }^{(29)}$.
Los/as participantes fueron enfermeros/as que se desempeñan en el RP, considerando como criterio de inclusión la experiencia laboral, superior a 6 meses, en el área penitenciaria al momento de la entrevista. Se utilizó el criterio de saturación constante para determinar el número de participantes, que en este caso fueron 6 profesionales de enfermería, siendo el $100 \%$ del área hospitalaria. El criterio de saturación se centra en finalizar la investigación cuando la producción de datos comienza a ser repetitiva y no aporta nueva información ${ }^{(29)}$. Lo relevante de este criterio es la diversidad de información que aporta cada uno de los participantes.

Para la producción de la información se utilizaron técnicas de entrevistas semiestructuradas, basado en un guion tópico de acuerdo con objetivos. Se seleccionó esta estrategia ya que era la más acorde a las características exploratorias del estudio. Por ello, se creó un guion de entrevista con dimensiones y preguntas orientadoras, permitiendo que, durante la entrevista, el equipo de investigación pudiese incorporar nuevas preguntas al guión resultante de la interacción con la entrevistada. Esta estrategia que se utiliza con el propósito de dar respuesta al objetivo de la investigación, permite determinar la profundidad de las dimensiones consideradas para el análisis de la entrevista ${ }^{(29)}$. El guión consideró las siguientes preguntas orientadoras: ¿Cuáles son las prestaciones de salud en el recinto penitenciario? ¿Cómo percibe usted el trabajo como enfermera/o en el contexto penitenciario? ¿Cuáles son las actividades que realizan las/os enfermeras/os? ¿Cómo describiría el contexto laboral en el área penitenciaria para el profesional de enfermería en particular? ¿Cuál es la labor de enfermería en el área asistencial, de gestión, educación e investigación en el contexto penitenciario? ¿Cuáles son las competencias necesarias para realizar esta labor en el contexto penitenciario? ¿Qué capacidades y/o actitudes ha tenido que desarrollar para desenvolverse en el contexto penitenciario? Durante las entrevistas no hubo limitantes ya que las/os enfermeras/os cooperaron contantemente durante el desarrollo de este proceso.

Para acceso al campo, se obtuvo autorización oficial de parte de las autoridades del RP. El desarrollo de las entrevistas se realizó en el lugar de trabajo, con un promedio de tiempo de una hora, en una sala privada. Los participantes firmaron 
consentimiento informado y los investigadores aplicaron la escucha activa que fue grabada mediante audio. La saturación de los datos se logró con un total de 6 personas entrevistadas.

Una vez realizado este proceso, se procedió a la transcripción literal, generando el corpus o texto de estas. Posteriormente, por medio del análisis de contenido, se realizó segmentación de la información para la creación de subcategorías, categorías centrales y generación de criterios de validez $^{(30,31)}$.

Del análisis de contenidos surgieron 15 subcategorías y 2 categorías, las que se procedieron a contrastar con el marco teórico, considerando citas textuales de las entrevistas como un elemento del contexto y así profundizar los hallazgos del trabajo de campo en la etapa de resultados.

La validación de la información se realizó mediante estrategia de triangulación de investigadores/as. Para ello el equipo de investigación realizó 6 reuniones de 90 minutos de duración, aproximadamente. En esta etapa también se consideró la revisión de la literatura, como criterio de contexto, lo que permitió generar discusión entre los integrantes del equipo, con el objetivo de evitar el sesgo de análisis y así cumplir con la triangulación entre diferentes investigadores/as.

Previo a que las/os participantes firmaran el consentimiento informado, se explicó el objetivo, beneficios y riesgos del estudio, enfatizando aspectos de confidencialidad ${ }^{(32)}$. El estudio posee resolución del comité de ética (069-9 agosto 2018), acreditado, de la Facultad de Medicina de la Universidad Católica del Norte ${ }^{(33)}$.

\section{RESULTADOS}

La/os profesionales de enfermería participantes presentan un promedio de experiencia clínica que fluctúa ente 3 a 7 años; para algunas/os ha sido una primera experiencia laboral en los RP, mientras que para otras/os ha sido una segunda opción laboral por experiencias previas a nivel hospitalario. Las dos categorías que se desprenden de los relatos de los profesionales y que le dan forma y sentido al análisis de contenido, son las siguientes:

\section{Realidad laboral de enfermería en el recinto} penitenciario: El "área laboral" corresponde al espacio donde las personas realizan diferentes funciones y presenta subcategorías: organización de equipos, reglamentos, protocolos, accesibilidad al campo, y percepción del clima laboral. La organización de equipos de salud del RP es multidisciplinaria y cada estamento cuenta con un sistema de turnos:

"Trabajan profesionales kinesiólogo, odontólogo, radiólogo, médico pediatra, ginecólogo, traumatólogo, matrona, equipo de enfermería y paramédicos. Son 11 paramédicos más una administrativa y 5 enfermeros. Cuatro en cuarto turno y una coordinadora. Un médico jefe que está cumpliendo con todas las horas"(E1).

Cada estamento profesional cumple con cierta cantidad de horas:

"Hay 4 enfermeras asistenciales, una coordinadora, un médico director y uno asistencial, 4 paramédicos en cuarto turno, 2 trabajan en cuarto turno modificado. Éstos trabajan largo, largo, libre, libre. Son los encargados de trasladar los medicamentos controlados y paramédicos que trabajan de jornada de 8 a 17 horas, uno de esterilización, dental y de farmacia" (E4).

"El kinesiólogo debe cumplir con 22 horas semanales, la matrona y el psiquiatra... por ejemplo yo, enfermera coordinadora tengo que cumplir con 45 horas semanales"(E1).

El personal de salud cuenta con "reglamentos internos", de vacaciones, permisos administrativos, permisos sin goce de remuneraciones por 3 meses por año, normativas para todos los profesionales:

"Nuestro sistema de vacaciones es igual que el resto de los trabajos, 15 dias hábiles anuales, más días administrativos que tenemos que son ocho. Bueno... igual es flexible, a veces podemos hacer cambios de turno, oh, por ejemplo, si hay alguna reunión importante a nivel de SEREMIA de algín programa se nos dan esos días libres" (E2).

La población del RP recibe atención de salud basada en protocolos asociados a empresas de capacitación, investigación y gestión en salud (CIGES), Asociación Chilena de Seguridad (ACHS) y Ministerio de Salud (MINSAL) y normas de la institución de Gendarmería, con los cuales deben dar cumplimiento a metas:

"Tenemos los protocolos del MINSAL y los que nos manda la ACHS que es nuestra empresa filial" (...) "todo lo que tenemos que cumplir" (E1).

"Si, claro, tenemos metas de cumplimiento algunas son 
guiadas por APS ejemplo, la cantidad de diabéticos y de hipertensos que tienen que estar compensados, pero si tenemos meta de EMPA y se debe 100\%"(E6).

El "acceso al campo laboral” está dado por la Empresa de Servicio de la Asociación Chilena de Seguridad (ESACHS) y es recomendable tener estudios y perfeccionamiento en urgencia y emergencia:

"ESACHS tiene una página de plataforma laboral, donde mandan ofertas de trabajo a todas las personas que hemos dado nuestro correo a la empresa, de hecho la empresa tiene un correo institucional" (E1).

"Acá si una persona que me dice que tiene el post título o un curso en urgencias, tendrá más prioridad que otra persona que no lo tenga" (E1).

El profesional de enfermería percibe un buen ambiente de "clima laboral" al interior del RP y destaca la seguridad durante sus jornadas laborales: "Siempre estamos acompañados de gendarmes, en hospitalización y atención en box...al atender se deja la puerta abierta, hay casos donde son pocos los gendarmes, asi que se pasean por fuera" (...) "el clima laboral es bueno, porque trabajamos pocas personas, es un trabajo regular, por ende no hay mayor complejidad" (E4).

El "área organizacional" es un conjunto de reglamentos y normas que regulan el funcionamiento de la Institución. Las subcategorías que emergieron fueron: prestaciones y demandas, derivaciones, evaluación de desempeño, infraestructura, paciente interno y atención de salud del paciente penitenciario. Las prestaciones en salud demandadas son: hospitalización básica para usuarios de ambos sexos, curaciones avanzadas, administración de medicamentos, exámenes de sangre, control de niño sano para lactantes hasta los 24 meses, consultas, controles, exámenes médicos preventivos de adultos y de adultos mayores.

En el caso de atención secundaria y/o terciaria de mayor complejidad o con riesgo vital se deriva al Hospital San Pablo de Coquimbo u Hospital San Juan de Dios de La Serena. Respecto a la atención primaria, el centro de salud familiar de derivación es Cardenal Caro:

"Nosotros si no tenemos resolución o si se necesita resolución secundaria se deriva, los médicos realizan interconsultas, tenemos una buena realimentación tanto con los hospitales y consultorios, que en este caso nuestra base es el Cardenal Caro...un paciente que necesite oftalmólogo se va al "UAPO"...si necesita control de pie diabético con un podólogo, nosotros tenemos comunicación con todos a nivel secundario" (E1).

"Pacientes más graves se derivan a hospital mediante carro fiscal de gendarmería o SAMU. Y se quedan alla”. (E4).

"Se estabilizan y los envian de vuelta para continuar con hospitalización acá". (E6)

El proceso de evaluación de desempeño es realizado por la enfermera coordinadora y médico director, así se visualiza en los siguientes relatos:

"Nosotros evaluamos, como enfermera coordinadora evalúo a mis paramédicos y enfermeros, y el médico director evalúa a los enfermeros incluyéndome a mi y a todo el equipo una vez al año (...) yo mensualmente mando las calificaciones de todos los paramédicos y se les dice a ellos en qué están fallando, en qué están bien" (E1).

La infraestructura del RP para la atención en salud consta de 16 camas para hospitalizados, algunas de estas están destinadas a aislamiento:

"Contamos camas para hospitalizado, son 16 camas 2 son de aislamiento para hombres y el resto son para damas y varones (...), en caso de que sea una mujer la que requiera aislamiento se habilita una de las cuatro camas de mujeres como aislamiento (E2).

El "paciente interno" es mayoritariamente masculino, presentan diversos trastornos psicológicos, en general respetuosos, conocen sus reglas y las respetan:

"Acá hay una población de 1780 reos aproximadamente diarios, de los cuales 190 son de mujeres aproximadamente (...) son pacientes diferentes a los del exterior, son manipuladores, fingen mucho. Uno tiene que saber diferenciar cuando mienten y cuando es real. Son respetuosos dentro de todo (...) las mujeres son más complicadas y más conflictivas”(E1).

La "atención de salud del paciente penitenciario" es el conjunto de situaciones de salud que presenta la población en el RP; la tuberculosis es una de las enfermedades que encabeza la lista, junto a heridas cortopunzantes por riñas masivas, enfermedades psiquiátricas y crónicas no transmisibles como dia- 
betes mellitus, hipertensión arterial, hipotiroidismo y epilepsia:

"Tenemos ahora a un paciente con observación de tuberculosis (...) otros pacientes están por neumotórax, hemoneumotórax, diabéticos descompensados, heridas penetrantes"(E4).

"Pacientes convulsionando, pacientes apuñalados, de todo" (E1).

"Tenemos pacientes de todo tipo, pacientes crónicos, descompensados con cáncer, curaciones, va a ver de todo"(E2).

\section{2. Área profesional de enfermería en los recintos} penitenciarios: El "área profesional" considera aspectos afines relacionados con el desarrollo profesional, de la cual se desprenden 5 subcategorías: experiencia y cargos, competencias, versatilidad, aprendizajes y funciones de Enfermería.

La experiencia de los profesionales de Enfermería es de 4 a 7 ańos aproximadamente; los cargos disponibles son coordinador/a, enfermero/a clínicos/as o asistenciales, más un enfermero/a clínico/a que se puede desempeñar como un subcoordinador subrogante:

"Yo como Enfermera coordinadora, fui enfermera de jornada de acá, el cual es un cupo que nunca antes se habia dado y que sólo se me dio a mi debido a mi post natal... entonces yo trabaje en la cárcel de Antofagasta en sistema de cuarto turno durante tres años y pedí traslado acá a la cárcel de Serena y por mi calidad de postnatal la empresa me creo este cupo especial que fue como enfermera de jornada en apoyo a mis colegas de cuarto turno"(E1).

"Enfermero asistencial y también a veces soy enfermero subcoordinador subrogante" (E2).

Entre las competencias generales deseables, para desempeñarse en los RP, destacan: madurez, paciencia y buen carácter:

"Madurez mental no tomar todo de forma personal. Hay que tener mucha paciencia, inteligencia emocional. No te pueden afectar las cosas que te dicen o se dicen entre ellos, no llevárselo a la casa. Mente plana sin prejuicios"(E6).

"Acá la persona debe ser una persona de buen carácter, o sea tú no tienes por qué gritarle a un paciente, no tienes por qué tratarlo mal (...) pero si un carácter fuerte porque si no te sobrepasan"(E1).

Los profesionales de enfermería consideran que su trabajo es versátil y les ofrece oportunidades de aprendizajes:

"Además tenemos atención de morbilidades" (E4).

"Es bastante transversal porque tenemos que ver desde el control de paciente crónico, controles de pacientes hospitalizados hasta atenciones de urgencias"(E2).

"Prácticamente todo lo aprendí en la unidad de salud, entonces todo, se ve urgencia, se tiene todos los programas que el gobierno exige, se tienen pacientes hospitalizados, es un mundo para aprender increible" (E1).

"Este es el lugar donde se aprende mucho, es una buena escuela (...) yo fui interna el 2011 y aprendi muchas cosas, porque no solo es procedimientos, sino que aprendes la toma de decisión, saber actuar rápido, es otro ambiente" (E6).

Las funciones del profesional de enfermería están relacionadas con tres áreas del quehacer: educación, gestión y asistencial. Las educaciones son diversas, en cuanto a grupos objetivos. Poseen planificación anual y mensual de capacitación para el personal, durante la atención se desarrollan educaciones básicas y obligatorias hacia la población penitenciaria:

"El enfermero que está a cargo del programa, dos o tres veces al año hace planes educativos, no tanto para el interno, sino para gendarmería y gente de aseo" (E1). "Son educaciones incidentales es que aqui no se pueden hacer educaciones grupales, por que corren mucho riesgo de peleas, es preferible atenderlos de forma individual, porque si yo tengo un grupo de internos significa que debo tener cierta cantidad de gendarmes" (E5).

Un 70 a $80 \%$ de las actividades son del área de gestión; como enfermero clínico debe estar a cargo de todo: coordinación con gendarmería, derivaciones externas, gestionar horas con los profesionales del centro de salud penitenciario, realizar reuniones con CIGES, además de estar atento sobre las gestiones de los pacientes hospitalizados:

"Acá todos tenemos nuestras funciones definidas y yo gestiono reuniones con SEREMIA, atenciones con el personal de salud, acá tenemos internos que son trabajadores de CIGES que trabajan en lavandería, reposteria, mantención, entonces ellos, sus jefaturas me mandan correo que necesitan atención entonces yo tengo que gestionar esas horas, que los programas se cumplan, que todo estébien en el piso de hospitalizados. 
Los enfermeros clínicos son los dueños del turno, entonces cualquier cosa, cualquier paciente que quieran derivar, yo tengo que preguntar al enfermero, porque él es quien coordina sus atenciones" (E1).

"Somos el nexo de casi todo... debemos hacer derivaciones, generar cosas con lavandería, cocina, con el hospital externo, con las urgencias, horas médicas a controles, salidas programadas" (E2).

Para dar cumplimiento a las normas ministeriales, el trabajo se organiza de acuerdo a programas: "Nos dividimos los programas...yo especificamente tengo a cargo el programa de epilepsia y supervisión. Otro enfermero tiene a cargo tuberculosis, epilepsia, otro cardiovascular, adulto mayor, otro enfermero tiene niños, porque acá hay niños hasta los 2 año" (E4).

Los enfermeros coinciden en que el contexto penitenciario no favorece el desarrollo de investigación, por la alta demanda asistencial y el estrés del ambiente laboral, sin embargo, manifiestan que se podría obtener información enriquecedora tanto para la profesión como para los usuarios:

"De hecho el área de investigación en enfermería no se ve mucho, la verdad es que con este cargo el tiempo es súper acotado, entonces no se puede" (E1).

En el área asistencial se realizan diversos procedimientos, desde control de signos vitales hasta curaciones avanzadas; las actividades son similares a un Centro de Salud Familiar:

"Tomamos exámenes de sangre, realizamos curaciones avanzadas, administramos tratamiento, realizamos atenciones de urgencias a pacientes que llegan graves ya que hay hartas riñas en la cárcel y debemos atender todas esas riñas, que más... realizamos EMPA, examen de medicina preventiva, control de pacientes crónicos del programa de salud cardiovascular, control de pacientes epilépticos, eh... que más... control de niño sano, mm... aplicación de EFAM, control de adultos mayores y morbilidades comunes, ah... $y$ hospitalizados, tenemos que ver hospitalizados también...tenemos una hoja diaria de atención de enfermería y lo tratamos como si fuera un servicio hospitalario con control de signos vitales, se realizan ingresos de enfermería" (E2).

El profesional de enfermería señala que la experiencia en los RP es una oportunidad para desempeñarse con autonomía, una instancia de aprendizaje y además le permite desarrollar competencias específicas derivadas de la demanda del contexto. Entre las motivaciones para desempeñarse en los recintos penitenciarios destaca la versatilidad del trabajo y seguridad de este:

"Me gusta el trabajo de enfermería, debes tener conocimientos, tomar decisiones, y saber actuar. Tienes cierta autonomía que a veces no a todos les acomoda en este contexto" (E6).

"Que en realidad yo lo encuentro bastante entretenido, es un trabajo que da una muy buena experiencia" (E2).

"Tenemos las urgencias que en mi opinión es lo más entretenido...depende de cada persona, para algunos trabajar con internos es estresante, pero para mi no lo es, además de contar con un gendarme, eso me da seguridad" (E4).

"Se te van todos los prejuicios, porque uno dice yo me levanto para atenderlo y para escucharlo, muchas veces son mentirosos y uno se ríe con ellos, pero pasan a ser también parte de lo que uno es, porque tú dices estoy salvándole la vida a un paciente" (E1).

\section{DISCUSIÓN}

De la información recopilada en este estudio, surgieron categorías que se relacionan con la "realidad laboral" y el "área profesional" de enfermería en los recintos penitenciarios, lo que permite describir las experiencias vivenciales de los profesionales que se desempeñan en este contexto.

El RP concesionado en la zona geográfica del norte de Chile, de acuerdo a este estudio, en el área de salud posee infraestructura y un equipo de salud multidisciplinario que garantiza las prestaciones de salud a la población. Coincide con Rodríguez y Tolosa ${ }^{(9)}$, quienes mencionan que "establecimientos penitenciarios deben garantizar el bienestar, seguridad, y preservación de la salud de las personas, independientemente de su condición”. Esto también lo señalan los convenios internacionales de los derechos humanos ${ }^{(20)}$ y concuerda con lo que describe Carrasco ${ }^{(10)}$ : "Se debe garantizar las prestaciones sanitarias de los internos como usuarios de los servicios de salud, la accesibilidad de este colectivo a los recursos sanitarios, la calidad de las prestaciones sanitarias penitenciarias y la equidad en el acceso a los cuidados". 
Así, el RP se convierte en un medio propicio para desarrollar el rol de enfermería. Su administración debe brindar salud de calidad y para ello se requiere de profesionales de enfermería que se rijan por normas y protocolos específicos que determinan las acciones a seguir y los procesos de evaluación que dan seguridad de la atención a los usuarios. La atención de salud debe responder a las orientaciones y políticas emanadas de la autoridad sanitaria, Ministerio y Servicios de Salud, considerando aspectos administrativos, técnicos, protocolos, procedimientos de traslados y derivaciones, autorizaciones sanitarias de unidades de salud, trabajo intersectorial, sistemas de registro y estadísticos, todos aspectos descritos en el manual de derechos humanos de la atención penitenciaria $^{(20)}$.

Considerar la atención y garantías universales en salud para todas las personas, independiente de su condición, es un desafío para el campo de enfermería en el contexto penitenciario en Chile, y es una realidad validada por los relatos profesionales que participaron en esta investigación.

También se debe recalcar que las experiencias de los profesionales de enfermería en estas instituciones es poco conocida y diferente de la atención llevada a cabo en el medio externo ${ }^{(4)}$, su labor en prisiones, por los condicionantes del contexto penitenciario, como son la privación de libertad del recluso y las influencias legales dentro del medio, necesita de un proceso de adaptación y capacitación específico. En el contexto penitenciario, brindar cuidados requiere de un apropiado clima laboral ${ }^{(33)}$, que sea percibido como seguro por los/as enfermeros/as, por la necesidad de enfrentar de mejor forma el estrés del contexto.

Respecto a las demandas de atención de salud más frecuentes encontradas en este estudio por patologías como la tuberculosis, es importante considerar a Fabrini et al. ${ }^{(13)}$, quienes señalan que las condiciones de encarcelamiento favorecen la transmisión de este proceso infeccioso. Las otras enfermedades presentes, como las psiquiátricas, crónicas y las heridas penetrantes, por riñas masivas concuerdan con lo descrito por Osses y Riquelme ${ }^{(14)}$, quienes plantean que un $45 \%$ de la población penal posee al menos una enfermedad diagnosticada, primando los desórdenes mentales, patologías del sistema respiratorio y circulatorio. En la misma línea, Barbosa et al. ${ }^{(15)}$ mencionan que existe una alta prevalencia de enfermedades infecciosas en el ámbito carcelario, importantes desde el punto de vista epidemiológico, como sífilis, hepatitis $\mathrm{B}$, tuberculosis y neumonía. Sanhueza y Candia ${ }^{(3)}$ también mencionan que la población privada de libertad tiene mayores prevalencias de enfermedades de transmisión sexual, crónicas o enfermedades de salud mental, y que la mayor prevalencia está dada por patologías infecciosas como el virus de la inmunodeficiencia humana (VIH), el virus de la hepatitis C (VHC), la tuberculosis y la hepatitis B y C.

En el área profesional, las funciones de enfermería que se desprenden de esta investigación se desarrollan principalmente en tres áreas: asistencial, educación y gestión. Esto es concordante con lo que señalan Karaaslan y Aslan ${ }^{(16)}$, quienes plantean que la/el enfermera/o debe resolver problemas y brindar orientación en actividades derivadas de la asistencia, gestión y educación traducidas en corregir malos hábitos como el consumo de alcohol, cigarrillos y drogas.

Por otro lado, las prestaciones de servicios en los RP, que son de nivel primario y secundario, se caracterizan por ser versátiles y dificultosas, tal como lo observa la American Nurses Association $(A N A)^{(5)}$ que considera que el trabajo de enfermeros/as en prisiones consiste en cuidar a personas con problemas de salud complejos.

En lo que respecta a educación en salud, esta es adaptativa ${ }^{(20)}$ al contexto, usuario y tiempo y se entrega a la población penal, al personal del servicio y al personal de Gendarmería.

En esta investigación, también se evidencia que un rol importante es el de la gestión. De los relatos de las enfermeras/os se desprende que asumen un rol gestor importante y de alta responsabilidad en la organización, tomando cargos fundamentales para el adecuado funcionamiento. Según Carrasco ${ }^{(10)}$ en la función de gestión y organización de la enfermería penitenciaria, se asume un trabajo administrativo de organización sanitaria, ratificado, por ejemplo, en la aplicación de protocolos emanados desde el Ministerio de Salud y Gendarmería.

La mayoría de los profesionales de enfermería coincide en que es un campo en donde se adquieren muchas habilidades, con oportunidades de aprendizaje en todas las áreas de enfermería y de ejercer autonomía en la toma de decisiones. Por ello se convierte en un contexto laboral recomendado 
ampliamente para futuros colegas, sin antes recibir capacitación en tanto en áreas específicas como salud mental, abuso de drogas, emergencias, atención primaria de salud y salud pública, como también en aspectos legales y regulatorios ${ }^{(12)}$.

Entre las limitaciones del presente estudio se pueden mencionar el acceso limitado a recintos penitenciarios y los tiempos limitados de los profesionales de enfermería para el desarrollo de las entrevistas. Por ello se deben realizar gestiones previas con las autoridades para obtener los permisos correspondientes.

\section{CONCLUSIONES}

Las experiencias de los profesionales de enfermería en el contexto penitenciario posee particularidades debido al contexto en el cual de desarrolla. Entre estas se encuentran las características que presentan los usuarios, las demandas y patologías prevalentes que requieren atención de salud, el proceso de adaptación de la cultura penitenciaria, el perfeccionamiento y el sistema de normas, reglamentos y protocolos que rigen sus funciones y el cumplimiento de su rol.

De las dos categorías que surgieron, la primera, relacionada con "area laboral", la constituyen los equipos de salud, que al interior de los RP tienen carácter multidisciplinario y el recurso humano de enfermería está organizado con una directora, una subdirectora y enfermeras/os clínicas/os que prestan servicio en cuarto turno. Los cuidados de enfermería se rigen por las normativas y protocolos que emanan desde el Ministerio de Salud para la entrega de todas sus prestaciones y para realizar derivaciones de situaciones de mayor complejidad se encuentran coordinados con los diferentes establecimientos de la red asistencial.

El clima laboral es un aspecto importante al momento de evaluar la eficacia y eficiencia de las diferentes actividades que deben realizar y este estudio revela que su desempeño se desarrolla en un clima laboral seguro, por el apoyo que brinda Gendarmería en cada ocasión que lo amerite.

La segunda categoría, "aspectos propios del área profesional de enfermería”, para su desarrollo existe una clara definición de funciones y distribución de trabajo de acuerdo al cumplimiento con los diferentes programas que debe desarrollar y se considera recurso humano vital al interior de los RP, porque brinda múltiples prestaciones a los usuarios en diferentes etapas de su ciclo vital, en acciones derivadas de promoción, recuperación de la salud, desempeñando actividades relacionadas con el área asistencial, de gestión y de educación, vinculadas con la demanda que presenta la población privada de libertad.

Los profesionales de enfermería valoran la experiencia que se desarrolla en los RP, la consideran enriquecedora desde el punto de vista de los cuidados que brinda, de la autonomía profesional, destacando el desarrollo de cualidades como la empatía, paciencia, y actuar sin prejuicios, entre otros.

Finalmente, el desarrollo de esta investigación muestra las experiencias en un contexto desconocido para muchos, entrega una mirada situacional y personal de los profesionales de enfermería que se desarrollan en dicho ámbito. Sería conveniente que se pudiera ampliar el conocimiento de otras realidades y a futuro estudiar la posibilidad de acceso a campo clínico para los futuros profesionales que están en sus últimos años de formación.

\section{REFERENCIAS}

1. Barbosa ML, Medeiros SG, Chiavone FBT, Atanásio LLM, Costa GMC, Santos VEP. Ações de enfermagem para as pessoas privadas de liberdade: uma scoping review. Esc Anna Nery [Internet]. 2019 [citado 2 feb 2021]; 23(3): e20190098. Disponible en: http://dx.doi.org/10.1590/21779465-EAN-2019-0098

2. Wong I, Wright E, Santomauro D, How R, Leary C, Harris M. Implementing two nurse practitioner models of service at an Australian male prison: A quality assurance study. J Clin Nurs [Internet]. 2018 [citado 2 feb 2021]; 27(1-2): e287-e300. Disponible en: http://dx.doi.org/10.1111/jocn. 13935

3. Sanhueza GE, Candia J. Acceso a la atención sanitaria en cárceles chilenas: una mirada desde los internos. Rev Esp Sanid Penitenciaria [Internet]. 2019 Feb [citado 2 feb 2021]; 21(1): 5-11. Disponible en: https://scielo.isciii.es/pdf/sanipe/ v21n1/es_1575-0620-sanipe-21-01-5.pdf

4. Sánchez-Roig M, Coll-Cámara A. Prison nursing and its training. Rev Esp Sanid Penit [Internet]. 2016 Dic [citado 13 abr 2018); 18(3): 110-118. Disponible en: http://dx.doi.org/10.4321/S1575- 
06202016000300005

5. American Nurse Association. The Correctional Nurse Educator [Internet]. 2013 [citado 29 may 2018]. Disponible en: https://www.correctionalnur seeducator.net/

6. Chaer-Y, Serroukh, S. Enfermería penitenciaria, la gran desconocida. Metas de enfermería [Internet]. 2018 Sep [citado 2 feb 2021]; 21(7): 62-66. Disponible en https://dialnet.unirioja.es/servlet/ articulo? codigo $=6534823$

7. Silva PB, Barbosa ML, Oliveira LV, Costa GM. Nursing care provided to persons deprived of liberty in the hospital environment. Rev Bras Enferm [Internet]. 2020 Abr [citado 1 feb 2021]; 73(3): e20170809. Disponible en: http://dx.doi. org/10.1590/0034-7167-2017-0809.

8. Zulaika D, Etxeandia P, Bengoa A, Caminos J, Arroyo-Cobo J. Un nuevo modelo asistencial penitenciario: la experiencia del País Vasco. Rev esp sanid penit [Internet]. 2012 Sep [citado 11 may 2018]; 14(3): 91-98. Disponible en: http://scielo. isciii.es/pdf/sanipe/v14n3/04_especial.pdf

9. Rodríguez S, Tolosa D. Viaje de ida y vuelta: Reflexiones sobre la enfermería psiquiátrica penitenciaria. Cultura de los Cuidados [Internet]. 2018 Jun [citado 19 may 2019]; 22 (52): 40-52. Disponible en: https://rua.ua.es/dspace/bitstream/ 10045/85309/1/CultCuid_52-40-45.pdf

10. Carrasco-Baún H. Prison nursing: legal framework and care reality. Rev esp sanid penit [Internet]. 2017 Jun [citado 11 may 2018]; 19(1): 3-12. Disponible en: https://scielo.isciii.es/pdf/sanipe/ v19n1/02_especial.pdf

11. Soares AAM, Castro GMO, Almeida IEM, Monteiro LAS, Torres LM. Vivências da equipe de enfermagem no cotidiano do sistema penal. Rev baiana enferm [Internet]. 2020 [citado 7 feb 2021]; 34: e34815. Disponible en: http://dx.doi. org/10.18471/rbe.v34.34815

12. González-Gálvez P, Sánchez-Roig M, Coll CA, Canet VO, Roca LJ. Ethical conflicts in nursing care in the prison context. Rev esp sanid penit [Internet]. 2018 Sep-Dic [citado 25 may 2018]; 20(3): 95-102 Disponible en: http://scielo.isciii. es/pdf/sanipe/v20n3/1575-0620-sanipe-20-03-95. pdf

13. Fabrini V, Carvalho B, Mendonça F, Guariente M. Care for people deprived of freedom with tuberculosis and permanent education in health. Trab educ saúde [Internet]. 2018 Sept-Dic [citado 11 abr 2019]: 16(3): 1057-1077. Disponible en: http://dx.doi.org/10.1590/1981-7746-sol00166

14. Osses-Paredes C, Riquelme-Pereira N. Situación de salud de reclusos de un centro de cumplimiento penitenciario, Chile. Rev esp sanid penit [Internet].
2013 Mar [citado 14 may 2018]; 15(3): 98-104. Disponible en: http://scielo.isciii.es/pdf/sanipe/ v15n3/03_original2.pdf

15. Barbosa L, Celino S, Oliveira L, Pedraza D, Costa G. Primary health care of convicts in the penitentiary system: subsides for nursing performance. Esc Anna Nery [Internet]. 2014 Dic [citado 2 jun 2018]; 18(4): 586-592. Disponible en: http:// dx.doi.org/10.5935/1414-8145.20140083

16. Karaaslan A, Aslan M. The Relationship Between the Quality of Work and Organizational Commitment of Prison Nurses. J Nurs Res [Internet]. 2019 Jun [citado 1 feb 2021]; 27(3): e25. Disponible en: http://dx.doi.org/10.1097/jnr.0000 000000000286

17. Chassagne A. Un regard ethnographique sur l'activité des infirmières dans une maison d'arrêt. An ethnographic look at the activity of nurses in a remand centre. Soins [Internet]. 2020 May [citado 2 feb 2021]; 65(845): 14-19. Disponible en: http:// dx.doi.org/10.1016/S0038-0814(20)30076-1.

18. Sanhueza G, Peréz F. Cárceles concesionadas en Chile: evidencia empírica y perspectivas futuras a 10 años de su creación. Polít crim [Internet]. 2017 [citado 15 may 2018]; 12(24): 1066-1084. Disponible en: http://dx.doi.org/10.4067/S071833992017000201066.

19. Espinoza O, Martínez F, Sanhueza G. El Impacto del Sistema Penitenciario en los Derechos Humanos: La Percepción de las Personas Privadas de Libertad. En: Informe Anual Sobre Derechos Humanos en Chile [Internet]. Ediciones de la Universidad Diego Portales. Santiago de Chile. Facultad de Derecho; 2014 [citado 20 abr 2018]. p. 245-289. Disponible en: https://derechoshumanos. udp.cl/cms/wp-content/uploads/2020/12/Cap-06Impacto-del-sistema-penitenciario-en-los-ddhhpercepcion-privados-libertad-1.pdf

20. Gendarmería de Chile. Manual de derechos humanos de la atención penitenciaria. Unidad de Protección y Promoción de los Derechos Humanos [Internet]. 2014 [citado 20 abr 2018]; p. 01-118. Disponible en: https://html.gendarmeria.gob.cl/ doc/ddhh/Archivos_Adicionales/MANUAL_ DDHH_GENCHI_FINAL.pdf

21. Espinosa A, Gibert M, Oria M. El desempeño de los profesionales de Enfermería. Rev Cubana Enferm [Internet]. 2016 Mar [citado 17 may 2018]; 32 (1): 87-97. Disponible en: http://scielo. sld.cu/pdf/enf/v32n1/enf10116.pdf

22. De Arco O, Suarez Z. Rol de los profesionales de enfermería en el sistema de salud colombiano. Univ Salud [Internet]. 2018 Abr [citado 30 may 2018]; 20(2): 171-182. Disponible en: https://doi. org/10.22267/rus.182002.121 
23. Muñoz L, Álvarez R, Cárcamo S, Espinoza S, Aguilar A, Morales V, et al. Tuning, Educación Superior en América Latina: Reflexiones y perspectivas en Enfermería [Internet]. Bilbao. Editorial Universidad de Deusto; 2013 [citado 1 jun 2018]. p. 90. Disponible en: www.deustopublicaciones.es/deusto/index.php/es/tuning-es/ tuning-america-es.

24. Arandojo M. Nuevas Tecnologías y nuevos retos para el profesional de enfermería. Index Enferm [Internet]. 2016 Jun [citado 5 abr 2018]; 25(1-2): 38-41. Disponible en: http://scielo.isciii.es/scielo. php? script $=$ sci_abstract\&pid $=$ S1 132-1296201 6000100009

25. Shamian J. El papel de la enfermería en la atención sanitaria. Rev bras enferm [Internet]. 2014 Dic [citado 14 mar 2018]; 67(6): 867-868. Disponible en: https://www.scielo.br/pdf/reben/v67n6/es_00 34-7167-reben-67-06-0867.pdf

26. Buitrago MLA, Arias LBE. Los aportes del enfoque biográfico narrativo para la generación de conocimiento en Enfermería. Index Enferm [Internet]. 2018 Jun [citado 13 may 2019]; 27(12): 62-66. Disponible en: http://scielo.isciii.es/ scielo.php?script=sci_abstract\&pid=S1132-12962 018000100013

27. Rubilar DG. Narrativas y enfoque biográfico. Usos, alcances y desafíos para la investigación interdisciplinaria. Enfermería (Montevideo) [Internet]. 2017 Oct [citado 14 may 2018]; 6(spe): 6975. Disponible en: http://dx.doi.org/10.22235/ ech.v6iespecial.1453
28. Flick U. Introducción a la investigación cualitativa. $2^{\mathrm{da}}$ ed. Barcelona: Ediciones Morata; 2012. 324p.

29. Mena L. La muestra cualitativa en la práctica: una propuesta. Revista eixo [Internet]. 2018 Dic [citado 3 jun 2019]; 8(3): 5-15 Disponible en: https://doi. org/10.19123/eixo.v8i3.646

30. Kallio H, Pietilä AM, Johnson M, Kangasniemi M. Systematic methodological review: developing a framework for a qualitative semi-structured interview guide. J Adv Nurs [Internet]. 2016 Jun [citado 2 feb 2021]; 72(12): 2954-2965. Disponible en: https://doi.org/10.1111/jan.13031.

31. Díaz C. Investigación cualitativa y análisis de contenido temático. Orientación intelectual de revista Universum. Rev gen inf doc [Internet]. 2018 Ene [citado 10 jun 2019]; 28(1): 119-142. Disponible en: https://dialnet.unirioja.es/servlet/ articulo? codigo $=6680164$

32. Suárez F. Un marco ético amplio para la investigación científica en seres humanos: más allá de los códigos y las declaraciones: la propuesta de Ezekiel J. Emanuel. Pers bioét [Internet]. 2015 Jul. [citado 15 abr 2018]; 19(2): 182-197. Disponible en: http://dx.doi.org/10.5294/PEBI.2015.19.2.2.

33. Contreras V, Reynaldos K, Cardona L. Clima, Ambiente y Satisfacción laboral: un desafío para la enfermería. Rev Cubana Enferm [Internet]. 2015 [citado 11 may 2018]; 31(1): [aprox. 0 p.]. Disponible en: http//www.revenfermeria.sld.cu/ index.php/enf/article/view/478 